\title{
INOVAÇÃO EM SERVIÇO: DESAFIOS E RESULTADOS EM PROJETOS DE ARQUITETURA
}

\author{
INNOVATION IN SERVICE: CHALLENGES AND PROJECTS IN ARCHITECTURE
}

\section{Sonia Regina Amorim Soares de Alcantara, M.Sc. (UFC) \\ Lucila Naiza Soares Novaes, Dra. (UFC) Thays Lyanny da Cunha Garcia da Rocha (UFC)}

\author{
Palavras Chave \\ Inovação; Empreendedorismo; Setor de Serviço; Projeto de Arquitetura
}

\section{Key Words}

Innovation; Entrepreneurship; Service sector; Architecture Project

\section{RESUMO}

A pesquisa está centrada no seguinte questionamento: como a inovação em serviço pode maximizar o resultado dos projetos de arquitetura? O objetivo geral é mapear impactos da inovação em serviços no desempenho dos projetos de arquitetura. O estudo justifica-se por buscar conhecer o impacto da inovação no ambiente da Arquitetura, inserida na importante cadeia produtiva da Indústria da Construção Civil, e com base neste conhecimento favorecer práticas direcionadas a uma melhor gestão de recursos e benefícios para a sociedade, consumidora final destes serviços. A Metodologia utilizada consiste em pesquisa de campo, descritiva, com abordagem qualitativa em uma amostra de empresas do Ceará filiadas ao Programa de Inovação da Indústria da Construção Civil (INOVACON) e à Associação Brasileira dos Escritórios de Arquitetura Regional Ceará (AsBEA-CE). Constatou-se que as inovações em serviços são complexas e acontecem nos últimos 20 anos em grande escala no âmbito da adoção de softwares, com destaque para a plataforma BIM (Building Information Modeling). Como resultado das inovações no processo de projeto tem-se projetos mais assertivos, criativos e focados nas necessidades dos clientes, execução facilitada, redução de custo e de tempo de execução.

\section{ABSTRACT}

The research is centered on the following question: how can in-service innovation maximize the outcome of architectural projects? The general objective is to map impacts of innovation on services in the performance of architectural projects. The study is justified by seeking to know the impact of innovation in the architecture environment, inserted in the important productive chain of the Construction Industry, and based on this knowledge favor practices aimed at a better management of resources and benefits for society. The methodology used is a descriptive field research with a qualitative approach in a sample of companies from Ceará affiliated with the Innovation Program of the Civil Construction Industry(INOVACON) and the Brazilian Association of Offices of Regional Architecture Ceará(AsBEA-CE). It was found that the innovations in services are complex and have occurred in the last 20 years in a large scale in the scope of software adoption, especially on the BIM (Building Information Modeling) platform. As a result of the innovations in the design process, we have more assertive, creative projects focused on customer needs, easier implementation, reduced cost and execution time, and broad compliance with the legislation. 


\section{INTRODUÇÃO}

O setor de serviços por ser dinâmico e possuir destacada importância para a economia e o desenvolvimento do país, permite que em sua essência exista inovação que, neste segmento, pode ser apoiada e desenvolvida em parceria com o consumidor final, o cliente. Autores como Schumpeter (1957), Freeman (1987, 1994) e Tidd (2001) destacam a importância da inovação para o desenvolvimento das empresas. Em consonância Vargas, Bohrer, Ferreira e Moreira (2013, p. 3), refletem sobre esta situação no Brasil, afirmando que hoje o setor já responde por mais de dois terços do Produto Interno Bruto e questionam a capacidade do setor de serviços de "contribuir para longos períodos de desenvolvimento econômico sustentável que permita a inserção e consolidação brasileira no rol das principais economias do mundo".

Além disso, com a competitividade crescente as empresas buscam melhorar a qualidade de suas ofertas agregando valor a elas. A inovação é opção a ser seguida por empresas para reter o cliente e se diferenciar no mercado. Nesse contexto, o tema da inovação significa algo novo, conforme discutido por diversos autores. (TIDD; BESSANT; PAVITT, 2001).

No âmbito da prestação de serviços em projetos de arquitetura, por estes serem os responsáveis pelos insumos mais estratégicos no resultado do processo de projeto, considera-se o processo de inovação essencial para atingir seu objetivo, que é valorizar, otimizar e atender as necessidades do cliente (CARVALHO, 2003). Portanto, esta pesquisa pretende responder à seguinte questão: Como a inovação em serviço pode maximizar o resultado dos projetos de arquitetura?

Em decorrência, o objetivo geral é mapear impactos da inovação no desempenho dos projetos de arquitetura. Especificamente, busca-se:

- Diferenciar o processo de inovação em produtos do processo de inovação em serviços;

- Levantar a ocorrência e a frequência de inovação no serviço de projeto de arquitetura;

- Identificar os impactos das inovações no processo de projeto de arquitetura.

$O$ estudo justifica-se porque permite conhecer o impacto da inovação no ambiente da Arquitetura, e com base neste conhecimento favorecer práticas direcionadas a uma melhor gestão de recursos e benefícios para a sociedade, consumidora final destes serviços.

A Metodologia utilizada consiste em uma pesquisa de campo, descritiva, com abordagem qualitativa em uma amostra de empresas do Ceará filiadas ao Programa de
Inovação da Indústria da Construção Civil (INOVACON), núcleo existente no Sindicato das Construtoras do Ceará (SINDUSCON-CE) e à Associação Brasileira dos Escritórios de Arquitetura regional Ceará (AsBEA-CE). A coleta de dados deu-se através de entrevista com profissionais representantes das empresas das respectivas instituições e da Universidade Federal do Ceará -UFC, contemplando as visões executiva e acadêmica.

Por fim, a análise de dados foi baseada na análise de conteúdo. A pesquisa contou com as seguintes etapas: levantamento de referencial teórico, definição da metodologia, análise de dados e considerações finais.

\section{O PROCESSO DE INOVAÇÃO NO EMPREENDEDORISMO EM SERVIÇOS}

Diante de um cenário mundial de competitividade e busca por diferenciais, a inovação torna-se necessária para que as empresas alavanquem seus resultados e mantenham-se no mercado (TIDD, 2001). Além de proporcionar estímulo ao desenvolvimento e oportunidades de ampliação da eficácia organizacional (TIDD; BESSANT; PAVITT, 2001).

Um conceito mais contemporâneo de inovação, pode ser o da Organização para a Cooperação do Desenvolvimento Econômico (OCDE) (2005, p. 55), que considera uma inovação "a implementação de um produto (bem ou serviço) novo ou significativamente melhorado, ou um processo, ou um novo método de marketing, ou um novo método organizacional nas práticas de negócios, na organização do local de trabalho ou nas relações externas".

Para chegar ao conceito acima, muitos estudos já foram implementados. Destaca-se que um dos maiores precursores no que tange a inovação relacionada aos negócios foi Schumpeter. Buscando investigar o que garantia o desenvolvimento dos negócios, o autor identificou que a inovação possuía grande impacto. Em sua obra "The Theory of Economic Devefopment", Schumpeter (1957), destaca que a inovação pode ocorrer de diferentes formas: através da inserção de um novo bem ou qualidade de bem, de um novo meio de produção ainda não testado, que não necessita ser baseado numa descoberta científica nova, a abertura de um novo mercado, no qual o ramo de atuação do produto ainda não tenha entrado, o alcance de novas fontes de matérias-primas, a fixação de uma nova organização, como exemplo, um monopólio.

Conforme Abernathy e Clark (1985) esse processo promove diferentes impactos na produção, tecnologia e mercado. Portanto, desenvolveram o conceito de 
transiliência, que consiste em como a inovação pode afetar a competitividade conforme afeta conhecimentos, recursos e habilidades já existentes nas organizações, com impactos diferentes na forma de produção e tecnologias.

Sobre os tipos de inovação, Tushman e Nadler (1986), reconhecem haver diferenças entre a inovação em produtos e a inovação em serviços. A de produtos ocorre quando há a fabricação de algo novo ou de processos, quando ocorre alguma alteração na maneira em que um produto é fabricado. A inovação em serviço ocorre na forma como um serviço é oferecido ou executado.

Freeman (1987) em sua concepção acerca da inovação, a divide em incremental, radical, de mudanças do sistema tecnológico e de mudanças no paradigma tecno-econômico (revolução tecnológica). Na inovação incremental podem ocorrer variações de intensidade constante seja no ramo de serviços ou indústria e geralmente surgem de programas de pesquisa e desenvolvimento e como resultado de melhorias sugeridas por profissionais ou usuários. A inovação pode ser resultado de uma solução criativa de um colaborador, uma nova forma de atender o cliente, uma alternativa de determinada etapa do processo produtivo ou a modificação de um insumo para o novo produto.

A inovação radical ocorre de forma descontínua e provém dos resultados de pesquisa e desenvolvimento. A inovação através de mudanças do sistema tecnológico alcança diversos setores da economia e resulta do cruzamento entre a inovação radical e incremental, juntamente com a organizacional. $E$ a de cunho tecno-econômico surge da combinação de inovações tecnicamente factíveis, afetando fortemente a estrutura da economia e as formas de produção. (FREEMAN, 1987).

De acordo com Tidd, Bessant e Pavitt (2001), o processo de inovação está associado à renovação e evolução do negócio, independentemente do porte da empresa. Nas grandes empresas este processo se dá por meio de áreas de pesquisa e desenvolvimento, e nas menores empresas, pelas soluções embasadas em experiências práticas de resolução de problemas.

Acerca da inovação em serviços, é necessário primeiramente caracterizá-los. Silva e Meireles (2008, p. 134) afirmam sobre o conceito de serviços que "é fundamentalmente diferente de um bem ou de um produto. Serviço é trabalho em processo, e não o resultado da ação do trabaIho; por esta razão elementar, não se produz um serviço, e sim se presta um serviço". Portanto, reafirma-se a diferenciação no processo de inovação em produto e em serviço, ante aos diferentes focos de cada um destes setores.
No que tange a literatura científica sobre inovação em serviços os estudos nessa área ainda são tímidos se comparados aos relacionados a produtos físicos, apesar do setor continuar em expansão. Conforme pesquisa realizada pela Confederação Nacional de Serviços (CNS, 2014), o maior índice de geração de empregos no Brasil é do setor de serviço.

Kon (2009. p. 298), corrobora a percepção das autoras e afirma que as inovações e mudanças nos setores produtivos e organizacionais acabaram por "acrescentar às atividades de serviços uma relevância crescente, não apenas como complementação das demais atividades produtivas, mas também como indutora do desenvolvimento econômico".

Referente ao processo inovação em serviços, autores como Gallouj e Weinstein (1997), Sundbo e Gallouj (1998) e Gallouj e Savona (2009) definem que as modificações presentes nesse processo podem ser de diferentes tipos:

- Melhoria - Inovação que aprimora o serviço e não altera a estrutura do sistema agregando valor das características finais, seja por competência ou caraterística técnica;

- Incremental - Altera a estrutura do sistema através da inserção de novos aspectos técnicos ou substituindo elementos anteriores, podendo envolver melhorias de características finais e otimização de custos, não alterando a estrutural geral do sistema.

- Recombinadora / Arquitetural - Inovação que expande as possibilidades a partir de novas combinações de aspectos finais e técnicas;

- Formalizadora - Ordenação de aspectos do serviço a fim de especificá-lo e concretizá-lo, dando-lhe uma forma;

- Ad hoc - Baseada na solução de problemas previamente definidos por demandas de clientes, utilizando informações para formular novas soluções e conhecimentos.

Desde Gallouj e Weinstein (1997) foram propostos modelos de inovação em serviço. Inicialmente abordando elementos de competência dos clientes, competência do prestador de serviço, tecnologia do prestador de serviço e o serviço final. Outros modelos foram aprimorados posteriormente com base no modelo de Gallouj e Weinstein (1997), são eles o modelo de Den Hertog, (2000), que consiste do alinhamento e interferência da comunicação nas relações de coprodução entre o cliente e o prestador do serviço; o modelo geral de De Vries (2006), adaptado com relação à adequação aos moldes de inovação em rede adotados pela sociedade; o modelo geral ampliado de Klement (2007), em que a inovação é classificada conforme as modificações nas características 
finais dos serviços; e o modelo das seis dimensões de Den Hertog et. al. (2010) que apresenta dimensões de novo conceito de serviço, nova interação com o cliente, novo sistema de valores/parceiros de negócios, novo modelo de receita, o novo sistema organizacional ou tecnológico de prestação de serviços.

Em suma, cada modelo apresenta melhorias na explicação do processo de inovação em serviços, apresentando ainda sim limitações devido ao ambiente macro e complexo da prestação de serviços e do mercado. Conforme revisão de literatura feita por Morrar (2014), a inovação em serviços vem adquirindo importante papel no desenvolvimento de economias mundiais. Assim, a seguir traz-se uma análise acerca da inovação no processo de projeto de arquitetura, um tipo de serviço prestado por empresas da área de Arquitetura que geram insumos para o mercado da construção civil.

\section{O PROCESSO DE PROJETO DE ARQUITETURA: A GERAÇÃO DE VALOR E A INOVAÇÃO}

Conhecer o processo de projeto de arquitetura e sua cadeia de valor é fundamental para se investigar como inovar nessa área e agregar valor a esse serviço. A identificação da cadeia de valor remete aos estudos de Porter (1985), que propõe um modelo acerca de como uma atividade de negócio recebe insumos (matéria prima), agrega valor a ela e entrega valor aos clientes na versão final do produto.

Dessa forma, é necessário olhar para o processo de projetos arquitetônicos, para identificar a sua cadeia de valor. Conforme Andrade, Ruschel e Moreira (2011), existem diversas formas de descrever o processo de projeto de arquitetura, porém pelas suas características, as definições são por vezes, inconclusivas, decorrente do fato deste tipo de projeto envolver criatividade como principal componente e pela complexidade e diversidade no processo, influenciada por aspectos como a natureza do projeto, as necessidades dos clientes, o perfil do projetista, dentre outros fatores.

O processo de projeto envolve uma sequência de atividades e habilidades para análise, síntese de informações e problemas, criatividade e avaliação para a busca de soluções adequadas para os problemas identificados e propostos para um projeto arquitetônico (FABRíCIO, 2002).

Segundo Liu, Oliveira e Melhado (2011), a criação do projeto de arquitetura é uma atividade complexa, executada pelos seguintes agentes: arquitetos e demais projetistas que traduzem as necessidades dos empreendedores em documentos, empreendedores que viabilizam a execução do projeto, construtores que constroem o que foi projetado, usuários que utilizam o serviço final, dentre outros intercessores.

É consenso entre os autores a relevância dos projetos no processo construtivo, e notadamente o projeto de arquitetura por ser a base de todos os demais projetos $\mathrm{e}$, principalmente, por ser estratégico na gestão do processo. É no projeto arquitetônico onde estão as decisões que influenciam no custo e, ao mesmo tempo, são potencialmente os maiores responsáveis pela geração de qualidade nas construções, além de serem os definidores dos espaços que influenciam o consumidor final (CARVALHO, 2003).

Assim, para obter qualidade na gestão dos processos que envolvem o projeto de arquitetura, destaca-se o início em razão das seguintes etapas: estabelecimento dos objetivos e parâmetros para o projeto; definição do escopo, conforme suas especialidades e etapas; planejamento dos recursos, prazos das diversas etapas e estabelecimento de cronograma (LIU; OLIVEIRA; MELHADO, 2011, p. 64).

Ainda no contexto da gestão do processo de projetos arquitetônicos, conforme Melhado et al. (2005), este envolve um conjunto de atividades de planejamento, organização, direção e controle do projeto e atividades estratégicas, como contratação de equipe para assegurar a qualidade do produto final, o projeto. "A gestão do processo do projeto é entendida como a administração que começa com uma ideia e finaliza com a produção de uma documentação (o projeto), cujos parâmetros geram a construção de um edifício" (FABRICIO; MELHADO, 2011, p. 64).

De acordo com Liu, Oliveira e Melhado (2011, p. 75), ocorre em muitos casos, a falta de integração direta entre a concepção do produto e o detalhamento de sua produção, durante o processo de projeto, assim "o relacionamento entre projetistas e construtoras muda conforme a complexidade do empreendimento". Com relação ao histórico de soluções para agregar valor ao processo do projeto de arquitetura têm-se alterações que saem de um modelo tradicional para um de construção enxuta (Lean Design), chegando ao estágio atual do BIM (Building Information Modeling ou Building Information Model). Segundo Anderser et. al. (2013) e Kowaltowski et. al. (2006), o processo denominado sequencial - tradicional é feito linearmente, ou seja, concepção dos arquitetos, concepção do projetista, elaboração de projetos complementares e execução da obra pelo construtor criados pela equipe de projeto.

Conforme Braz (2016, p.2-3) "os desenhos produzidos anteriormente, à mão, estão sendo executados também 
através da utilização do computador. Vários softwares são hoje utilizados na criação e desenvolvimento de projetos de arquitetura e urbanismo". A autora cita softwares como: AutoCAD e SketchUP, ArchiCAD, Revit, Grasshopper, Rinocerus. Destaca em especial aqueles ligados a Modelagem da Informação da Construção BIM, como uma "plataforma que integra vários softwares, causando uma revolução no processo produtivo da Arquitetura e das Engenharias" (BRAZ, 2016, p. 3).

Conforme Tzortzopoulus e Formoso (1999), essas mudanças foram necessárias para a melhoria do processo de projeto. Em estudos realizados por Dantas Filho (2016), verificou-se que mais de $70 \%$ do tempo do processo de projeto de arquitetura é tempo sem agregação de valor e impactam diretamente no tempo total, assim sendo necessário mapear e analisar o seu fluxo de valor para permitir melhorias do processo. Leite (2014) corrobora afirmando, que novas pesquisas em gerenciamento de projetos têm sido desenvolvidas e que estas consideram o contexto atual caracterizado por incertezas, alto grau de complexidade e a questão de valor sob o ponto de vista do cliente.

Na perspectiva do modelo Lean Desgin, "há a necessidade do aprendizado contínuo ao longo do processo, o que não ocorre no modelo tradicional" (LEITE, 2014, p. 35). O modelo BIM - Building Information Modeling, diferentemente do sistema de desenhos CAD -Computer Aided Design, não é apenas uma forma de representação do projeto final após sua criação, trata-se de uma simulação e utilização de modelagem computacional para a construção e operação do empreendimento em si (NASCIMENTO et al, 2012). Ou seja, caracteriza-se como uma ferramenta de desenvolvimento de projetos integrados no processo de construção do projeto, é, portanto, uma das principais formas de inovar na entrega e agregar valor ao cliente.

Conforme Abernathy e Clark (1985), para que a inovação seja eficiente é necessário que esteja embasada em alguma necessidade do cliente, pois não é coerente inovar e ter custos sem que seja atrativo e útil. Sendo este um processo que envolve elementos como aquisição e transferência de conhecimento. Os estudos de Lawson (2011) indicam que para agregar valor através de uma inovação, é necessário conhecer as necessidades do cliente, pensar como ele e colocar-se no seu lugar, como exemplifica: "... conhecer os movimentos de nado de peito não impede que alguém se afogue na piscina. Cada um terá de entender como aquilo funciona por conta própria" (LAWSON, 2011, p.48). Assim, para garantir a sobrevivência, as empresas devem elaborar projetos com soluções concretas, definir critérios de prioridades, promover ações e iniciativas de desenvolvimento sócio-econômico mediante formação interdisciplinar e multifacetada, para uma intervenção prática, funcional e positiva na sociedade (NOVAES, 2003).

Kowaltowski, Bianchi e Petreche (2011, p. 23), afirmam que "com a crescente complexidade no mundo do projeto, a inovação, ou o estímulo ao pensamento criativo, deve deixar de confiar no talento ou no acaso. Para muitos profissionais, a inovação é a meta principal, afastando a repetição de ideias".

\section{METODOLOGIA}

Para configurar a metodologia da pesquisa, aqui serão abordados aspectos referentes à caracterização do um estudo e sua delimitação dentro do campo científico. Conforme Vergara (2013) pesquisa consiste em buscar conhecer o novo através de questionamentos e investigações.

O estudo caracteriza-se como descritivo e de abordagem qualitativa, já que se propõe a mapear impactos da inovação em serviços no desempenho dos projetos de arquitetura. A pesquisa descritiva define "características de determinada população ou de determinado fenômeno" (VERGARA, 2013, p. 42).

A estratégia de pesquisa utilizada foi a pesquisa de campo, tendo em vista a atuação dos pesquisadores em campo junto a amostra escolhida. A técnica de coleta de dados foi entrevista.

$O$ instrumento de coleta de dados utilizado foi o roteiro de entrevista padronizada, com dezessete perguntas abertas. O Universo da pesquisa é composto por $28 \mathrm{em}$ presas filiadas ao Programa de Inovação da Indústria da Construção Civil (INOVACON), núcleo de inovação dentro do Sindicato das Construtoras do Ceará (SINDUSCON-CE), e 27 escritórios de arquitetura associados a Associação Brasileira dos Escritórios de Arquitetura Regional Ceará (AsBEA-CE). A amostra, por acessibilidade, resultou na entrevista a doze empresas, sendo seis do INOVACON e seis da AsBEA-CE. Além disso, foi entrevistado representante do segmento acadêmico, professor/doutor do Centro de Tecnologia da UFC, permitindo enriquecer a análise com a percepção do setor que presta o serviço e do setor que gera o conhecimento científico no uso da inovação nos projetos de arquitetura.

Para o tratamento das informações coletadas, foi utilizada a análise de conteúdo. Para Bardin (2006, p. 38), consiste em "um conjunto de técnicas de análise das comunicações, que utiliza procedimentos sistemáticos e objetivos de descrição do conteúdo das mensagens". 


\section{PESQUISA: DISCUSSÃO E RESULTADOS}

As entrevistas foram realizadas com 13 pessoas, conforme definido nos critérios da amostra: 06 Engenheiros Civil do INOVACON, 06 Arquitetos da AsBEA-CE, 01 Engenheiro Civil, professor/doutor e diretor do Centro de Tecnologia da UFC. As perguntas foram as mesmas para todos os participantes da pesquisa, a fim de viabilizar o estudo das percepções.

A análise dos dados está dividida em três etapas, conforme os objetivos da pesquisa. Portanto, a primeira está relacionada à diferenciação do processo de inovação em produtos do processo de inovação em serviço. A segunda etapa refere-se a ocorrência e a frequência de inovação no serviço de projeto de arquitetura e a última identifica os impactos das inovações no processo de projeto de arquitetura.

\subsection{Diferenciação do processo de inovação em} produtos do processo de inovação em serviço

A fim de conhecer melhor a visão dos entrevistados sobre o processo de inovação, indagou-se a eles se haveria diferença entre a inovação em produtos e a inovação em serviços, já que todo o estudo está centrado na temática da inovação. Dessa forma, todos os entrevistados afirmaram que o processo de inovação em produtos é diferente do processo de inovação em serviços no sentido de que demonstrar o valor da inovação em produtos tangíveis é mais compreendido pelo cliente, enquanto que inovar em serviços é gerar um valor a ser percebido, intangível, portanto, mais complexo. Conforme destacado no referencial por Tushman e Nadler (1986) a inovação entre produtos e serviços é diferente devido ao processo de elaboração de ambos. Também Andrade, Ruschel e Moreira (2011), reconhecem que o projeto de arquitetura pode variar pela complexidade e diversidade no processo, influenciada por aspectos como a natureza do projeto, as necessidades dos clientes, o perfil do projetista, dentre outros fatores, confirmando a diferenciação destacada pelos entrevistados.

Sobre como se caracterizam as etapas do processo de inovação no projeto de arquitetura os participantes em sua maioria citaram que a inovação está em todo o processo do projeto, desde a concepção até a finalização, porém, mais especificamente na concepção pela visão mais presente nas palavras dos entrevistados do INOVACON. Nesse aspecto o professor entrevistado também afirmou que a inovação pode surgir em todo o processo projetual, sendo mais intensa na concepção, pois é a fase em que são pensadas as principais soluções de todo o processo. Conforme referenciado por Tidd, Bessant e Pavitt (2001), o processo de inovação está associado à renovação e evolução do negócio, assim ela pode surgir em diversas etapas.

\subsection{Ocorrência e frequência de inovação no ser- viço de projeto de arquitetura}

Com relação ocorrência da inovação e sua frequência no processo de projeto de arquitetura, perguntou-se inicialmente para os entrevistados que inovações já foram utilizadas no processo de projeto de arquitetura.

Percebe-se que os arquitetos, representados pela AsBEA-CE e engenheiros pelo INOVACON, citaram mais fortemente softwares como principais inovações já utilizadas (AUTOCAD, REVIT e SKETCHUP), sendo que as inovações por outros meios foram menos citadas, como a utilização da maquete fisica e utilização de materiais pré-fabricados na obra. Ante a indagação sobre qual seria a principal inovação ocorrida no processo de projeto de arquitetura nos últimos 20 anos, 100\% da AsBEA-CE e do INOVACON, citaram o BIM. O posicionamento do professor foi também direcionado para o BIM, fundamentado no processo de mudança do desenho do projeto a mão para a evolução dos softwares, passando de um modelo 2D, que tem somente visualização plana, feito em CAD - Computer Aided Design, para dimensão 3D, que é a visualização do plano, profundidade e altura, feita na modelagem da informação - BIM. No sistema BIM é possível ainda, a visualização da obra de acordo com o cronograma, inclusive em estágios futuros no tempo, permitir inclusão de dados de especificações, custos, garantias, fornecedores dos elementos do projeto, dentre outros, qualificando o acesso à informação, aspectos fundamentais para a gestão dos resultados do negócio.

Conforme citado no referencial, o BIM ter aparecido com mais frequência pode ser justificado por Nascimento et. al. (2012), que o caracteriza como uma das principais formas de inovar na entrega e agregar valor ao cliente. Corrobora o que foi citado por Braz (2016), de que a forma de desenho do projeto manual foi transformada pela utilização de softwares, sendo que a plataforma BIM, é o mais revolucionário por integrar o projeto com vários outros softwares.

Ainda com relação a ocorrência da inovação no processo de projeto foi questionado aos entrevistados quais as principais barreiras / dificuldades para a implementação da inovação na atividade do processo de projeto de arquitetura. Constatou-se que, em sua maioria, tanto os filiados da AsBEA-CE como os filiados do INOVACON, apontam a cultura das pessoas de resistência a mudanças e a falta de mão-de-obra especializada para lidar com a 
inovação. Os arquitetos revelam ainda os seguintes aspectos dificultadores da inovação: dificuldade de passar a inovação para o cliente e a desvalorização do serviço de projeto de arquitetura.

A cultura das pessoas / resistência a mudanças é um dos aspectos mais citados por ambas categorias de entrevistados, seguida pela dificuldade em passar a inovação para o cliente. Isso pode ser relacionado com o que foi afirmado por Liu, Oliveira e Melhado (2011), que em muitos casos ocorre uma falta de integração direta entre a concepção do produto e o detalhamento de sua produção, fatores que exigem compreensão por parte do arquiteto, quem fornece o serviço de projeto, e o construtor, quem recebe esse serviço. Assim, as pessoas são parte fundamental nesse processo, o que pode ocasionar resistência por outros fatores com relação à implantação da inovação e adaptação a ela.

A resistência das pessoas a inovação também foi evidenciada na análise das desvantagens com relação a utilização da inovação, ante a afirmação da AsBEA-CE de que faltam profissionais capacitados para lidar com os softwares. Em sua maioria, INOVACON e AsBEA-CE afirmaram não haver desvantagem em adotar inovação em seus processos.

$E$, quanto a frequência do uso dessa inovação nos projetos todos os entrevistados arquitetos afirmaram que a inovação está presente em todos, na totalidade ou parcialmente implementada, e quatro engenheiros concordam com esse posicionamento, sendo que os outros dois afirmam que ela está presente em quase todos.

\subsection{Impactos das inovações no processo de pro- jeto de arquitetura}

No que tange a impactos no processo de projeto, avaliou-se a visão dos entrevistados com base no desempenho do projeto, maximização dos resultados, finalidades e principais melhorias já implementadas a partir da utilização da inovação.

Com relação aos aspectos positivos, percebe-se pelo menos cinco dos seis entrevistados de cada instituição citou a otimização do tempo, seguido de soluções mais precisas, modernas, corretas e que atendem mais normas projetuais. Isso pode ser justificado pelo posicionamento anterior de terem citado o BIM como a principal ferramenta de inovação dos últimos 20 anos. Sobre impactos negativos, os arquitetos citaram: Não conseguir implantar todas as inovações desejadas, postura resistente do arquiteto a se adaptar as inovações em ferramentas, resistência das pessoas, custos da inovação e, com relação aos engenheiros, citaram ainda os custos da inovação e apenas um citou a resistência das pessoas.

Questionou-se também aos entrevistados sobre como a inovação pode maximizar o resultado dos projetos de arquitetura.

As percepções dos entrevistados acerca da inovação como vetor de otimização dos resultados no projeto destacam: na visão dos arquitetos, estão centradas na utilização de ferramentas/softwares tecnológicos como forma de melhorar o processo e qualidade do projeto e, na visão dos engenheiros, na redução de custos/melhores resultados financeiros. Outras percepções de ambos profissionais estão relacionadas entre si e envolvem: melhor informatização do projeto/especificações. O posicionamento do entrevistado da UFC é de que a inovação proporciona maior capacidade para o arquiteto em desenvolver projetos mais arrojados, com mais detalhes de apresentação, que facilitam a compreensão do cliente. Essa percepção também está citada por ambos profissionais, arquitetos e engenheiros.

Também foi indagado aos entrevistados a finalidade da utilização da inovação no processo de projeto. Os entrevistados registraram, em sua maioria, que a inovação no processo de projeto destina-se a agregar valor ao projeto e a atender às necessidades dos clientes. Ainda nessa perspectiva, todos os participantes da AsBEA-CE afirmaram que a inovação nos projetos gerou ao final maior agregação de valor e satisfação do cliente, e todos os entrevistados do Sinduscon, afirmaram perceber essa agregação e estarem satisfeitos.

Essa discussão sobre a finalidade da inovação e seus impactos no processo de projeto pode ser justificada como citado no embasamento teórico por Schumpeter (1957), Freeman (1987, 1994) e Tidd (2001) de que a inovação é importante para o desenvolvimento das empresas. Neste cenário, verifica-se que tanto os fornecedores do serviço do projeto de arquitetura, como os compradores deste, empresas de construção civil, percebem que a inovação é para agregar valor.

A fim de consolidar as percepções colhidas anteriormente junto aos entrevistados, foi questionado aos entrevistados da AsBEA-CE se os clientes deles conseguiam perceber essa inovação e, aos do INOVACON, se eles conseguiam perceber as inovações geradas pelos arquitetos nos projetos. Nesse sentido, constatou-se que apenas quatro arquitetos afirmaram com certeza de que o cliente conseguia perceber a inovação e dois afirmaram que não. Na visão dos engenheiros todos afirmaram que conseguem perceber quando os arquitetos inovam e inclusive dois deles afirmaram que essas inovações são avaliadas em conjunto. 
E por fim, foi indagado aos participantes quais as principais melhorias já foram implementadas com relação ao processo de projeto de arquitetura. Essas melhorias são:

- AsBEA-CE - Conseguir trazer o desenho arquitetônico para o computador, através de ferramentas e softwares e hardwares, eliminando perdas de informações; algumas melhorias foram: ganho qualitativo nas decisões do projeto, minimização de perdas e conflitos, diminuição de tempo projetual; utilização de softwares para verificação de inconformidades; melhoria dos processos, com controles mais precisos e organização da equipe de trabalho; os softwares proporcionam fidelidade ao objeto construído, rapidez, compreensão e qualidade do produto final; os softwares propiciam melhor visualização do projeto, de forma mais precisa em diversos ângulos do observador.

- INOVACON - Qualidade e precisão, e melhor clareza nas informações; redução de desperdícios e consequente resultado financeiro melhor, possibilidade de desenvolver um volume maior de projeto para as obras; antecipação de problemas, melhor visualização do projeto em softwares, soluções mais adequadas; os softwares permitem a execução de modelagem e a redução do tempo de entrega; os softwares propiciam maior integração das informações e melhor qualidade no detalhamento do projeto.

Constata-se que a utilização dos softwares permite a melhoria mais citada entre os entrevistados, a partir da exploração de suas variadas funções que proporcionam integração e segurança de informações, melhor visualização do projeto, controles mais precisos, otimização do tempo, redução de despesas/desperdícios e a qualidade no detalhamento do projeto.

Essa situação pode ser reforçada a partir do que foi destacado por Tidd, Bessan e Pavitt (2001), de que a inovação, além de proporcionar estímulo ao desenvolvimento, também gera oportunidades de ampliação da eficácia organizacional.

\section{CONCLUSÃO}

Conforme descrito no referencial, a inovação é uma das formas de maximizar os resultados das organizações e proporcionar um diferencial competitivo. Nesse contexto, esta pesquisa teve como questionamento como a inovação em serviço pode maximizar os resultados no projeto de arquitetura.

Com relação ao objetivo geral da pesquisa constatou-se que os principais impactos da inovação no desempenho dos projetos de arquitetura estão diretamente ligados a utilização de softwares, através da plataforma BIM.

Acerca da diferenciação do processo de inovação em produtos do processo de inovação em serviços constatou-se que, a maioria dos entrevistados, têm consciência de que o processo é distinto, mais difícil no âmbito dos serviços em face da sua complexidade, e pode acontecer durante todo o processo de projeto, evoluindo com sua execução.

No que tange a ocorrência e a frequência de inovação no serviço de projeto de arquitetura, nos últimos vinte anos, a maior inovação no processo de projeto foi o uso de softwares para substituir o desenho manual, com maior versatilidade para a criação e aplicação dos projetos, e a modelagem de informação da construção - BIM é atualmente uma plataforma que integra softwares tornando-se a mais recente inovação. Nestes vinte anos, o cenário demonstra grande ocorrência de inovação no serviço de projeto de arquitetura, o que é corroborado pelos arquitetos e engenheiros entrevistados, que afirmaram introduzir inovações frequentes em seus projetos. Estes profissionais citaram como principais dificuldades para adotar inovação: cultura de resistência a mudanças, dificuldade em demonstrar inovação para o cliente e escassez de mão de obra qualificada para a aplicação das inovações.

Os impactos da inovação no processo de projeto são projetos mais assertivos, criativos e focados nas necessidades dos clientes, execução facilitada, redução de custo e de tempo de execução. E sobre impactos negativos, os mais citados foram resistência das pessoas e custos da inovação.

Acredita-se que os objetivos da pesquisa foram alcançados e que ela pode ser insumo para elaboração de novos estudos que venham somar conhecimentos para a gestão da inovação em serviços e de projetos de arquitetura, ampliando assim, a comunicação entre os membros da cadeia produtiva da inovação no segmento de serviços.

\section{REFERÊNCIAS}

ABERNATHY, W. J.; CLARK, K. B. Innovation: Mapping the Winds of Creative Destruction. Research Policy, Amsterdam, v.14, n.1, p. 3-22, Feb. 1985.

ANDERSEN, M. et al. Identifying and modeling the integrated design process of net Zero Energy buildings. In: High Performance Buildings-Design and Evaluation Methodologies. 2013.

ANDRADE, M. L. V. X.; RUSCHEL, R. C.; MOREIRA, D. C. 0 processo e os métodos. In: KOWALTOWSKI, Doris C.C. K., MOREIRA, Daniel de Carvalho, PETRECHE, João R. D., 
FABRICIO, Márcio M. (orgs.). O processo de projeto em arquitetura. São Paulo: Oficina de Textos, 2011.

BARDIN, L. Análise de conteúdo. Lisboa: Edições 70, 2006.

BRANDÃO, O. C. S. Sobre fazer projeto e aprender a fazer projeto. Tese (Doutorado em Projeto de Arquitetura) - Faculdade de Arquitetura e Urbanismo, Universidade de São Paulo, São Paulo, 2008.

BRAZ, Z. L. Novas mídias no ensino de arquitetura e urbanismo: relação entre tecnologias, espaço e pedagogia. Dissertação (mestrado) - Universidade Federal de Viçosa.Viçosa, MG, 2016.

CARVALHO, A. C. N. Uma análise da relação entre o escritório de arquitetura e a pequena e média Construtora sob a ótica da parceria na gestão do empreendimento imobiliário. Dissertação (Mestrado em Administração) - Administração, Universidade Estadual do Ceará, Fortaleza, 2003.

DANTAS FILHO, J. B. P. Oportunidades de melhoria no processo de projeto de arquitetura sob a perspectiva do Lean Design. 2016. Dissertação (Mestrado em Engenharia Civil: Estruturas e Construção Civil) Centro de Tecnologia, Universidade Federal do Ceará Fortaleza, 2016.

DEN HERTOG, P. Knowledge-Intensive Business Services as Co-Producers of Innovation. International Journal of Innovation Management , 491-528, 2000.

DEN HERTOG, P.; VAN DER AA, W.; DE JONG, M. W. Capabilities for managing servisse innovation: towards a conceptual framework. J. Service Management, v. 21, n. 4, p. 490- 514, 2010.

FABRICIO, M. M.; MELHADO, S. B. O processo cognitivo e social do projeto. In: KOWALTOWSKI, Doris C.C. K., MOREIRA, Daniel de Carvalho, PETRECHE, João R. D., FABRICIO, M. M. (orgs.). O processo de projeto em arquitetura. São Paulo: Oficina de Textos, 2011.

FABRICIO, M. M. Projeto Simultâneo na construção de edifícios. Tese de Doutorado, Escola Politécnica, Universidade de São Paulo, 2002, 329p.
FREEMAN, C. Innovation and Growth. In: ROTHWELL, Roy; DODGSON, Mark (eds.). The Handbook of Industrial Innovation. Cheltenham: Edward Elgar Publishing, p.7893, 1994.

FREEMAN, C. Technology policy and economic performance. Londres: Pinter Publishers London and New York, 1987.

GALLOUJ, F. Innovating in reverse: services and the reverse product cycle. European Journal of Innovation Management, v. 1, n. 3, p. 123-138, 1998.

GALLOUJ, F.; SAVONA, M. Innovation in services: a review of the debate and the research agenda. Journal of Evolutionary Economics, v. 19, n. 2, p. 149-172, 2009.

GALLOUJ, F.; WEINSTEIN, O. Innovation in services. Research Policy, n. 26, p. 537-556,1997.

KIATAKE, M. Modelo de Suporte ao Projeto Criativo em Arquitetura: uma aplicação da TRIZ - teoria da solução inventiva de problemas. Dissertação. Escola Politécnica da Universidade de São Paulo, São Paulo, 2004.

KLEMENT, C. F. F. Inovação em serviços: estudo de casos em uma organização da indústria hoteleira brasileira. Tese (Doutorado em Administração). 141p. Universidade de São Paulo. São Paulo: 2007.

$\mathrm{KON}, \mathrm{A}$. O novo regionalismo e o papel dos serviços no desenvolvimento: transformações das hierarquias econômicas regionais. Revista OIKOS, v. 8, n. 2, pp.279300, 2009.

KOWALTOWSKI, D. C.C. K.; BIANCHI, G.; PETRECHE, João R. D. A criatividade no processo do projeto. In: KOWALTOWSKI, Doris C.C. K., MOREIRA, Daniel de Carvalho, PETRECHE, João R. D., FABRICIO, Márcio M. (orgs.). O processo de projeto em arquitetura. São Paulo: Oficina de Textos, 2011.

KOWALTOWSKI, D. C. C. K. et al. Reflexão sobre metodologias de projeto arquitetônico. Ambiente Construído, Porto Alegre, v. 6, n. 2, p. 7-19, abr./jun. 2006.

LAWSON, B. Como arquitetos e designers pensam. Tradução de Maria Beatriz Medina. São Paulo: Oficina de textos. 2011. 
LEITE, K. P. Proposta de melhorias do processo de projeto e de desenvolvimento de produtos em empreendimentos imobiliários. 2014. 229 f. Dissertação (Mestrado em Engenharia Civil: Estruturas e Construção Civil) - Centro de Tecnologia, Universidade Federal do Ceará, Fortaleza, 2014.

LIU, A. W. OLIVEIRA, L. A.; MELHADO, S. B. A gestão do processo de projeto em arquitetura. In: KOWALTOWSKI, Doris C.C. K., MOREIRA, Daniel de Carvalho, PETRECHE, João R. D., FABRICIO, Márcio M. (orgs.). O processo de projeto em arquitetura. São Paulo: Oficina de Textos, 2011.

NOVAES, L. N. S. A Evolução da Atuação Empreendedora dos Arquitetos na Cidade de Fortaleza. Dissertação mestrado, curso Administração na Universidade Estadual do Ceará, Fortaleza-CE, 2003.

NASCIMENTO, E. L.; BIZ, A. A.; FREITAS, M. C. D.; SCHEER, S. Modelagem de Informações no Desenvolvimento Enxuto de Projetos. In: SALGADO, M. S. et al. Projetos Complexos e seus Impactos na Cidade e na Paisagem. Rio de Janeiro: UFRJ/FAU/PROARQ; ANTAC, 2012.

OCDE. Organização para a Cooperação e Desenvolvimento Econômico \& Financiadora de Estudos e Projetos. Manual de Oslo. (3a ed.). 2005. Acesso em 24 abr, 2017. Disponível em:<http://www.mct. gov.br/upd_blob/0011/11696.pdf.>.

PORTER, M. E. The Competitive Advantage: Creating and Sustaining Superior Performance Simon \& Schuster, 1985.

SCHUMPETER, J. A. The theory of economic development. Cambridge, Harvard University. 1957.

SILVA E MEIRELLES, D. Serviços e desenvolvimento econômico: características e condicionantes. Revista de Desenvolvimento Econômico. n. 17, p. 23- 35, Jan. 2008.

SUNDBO, J.\& GALLOUJ. F. Innovation in services. Services in innovation, innovation in services. (SI4S Topical Paper, n.4). Oslo, Noruega, 1998.

TIDD, J. Innovation Management in Context: Environment, Organization and Performance. International Journal of Management Reviews, v. 3, n. 3, p. 169-183, Sep. 2001.
TIDD, J.; BESSANT, J.; PAVITT, K. Managing innovation: integrating technological, market and organizational change. Chichester: John Wiley \& Sons, 2001.

TUSHMAN, M.; NADLER, D. Organizing for innovation. California Management Review, Berkerley, v. 28, n. 3, p. 74-92, 1986.

TZORTZOPOULOS, P.; FORMOSO, C. Considerations on application of lean construction principles to design management. Proceedings IGLC, v. 7, p. 26-28, 1999.

VARGAS, E. R.; BOHRER, C.; FERREIRA; L. B.; MOREIRA, $M$. F. A pesquisa sobre inovação em serviços no Brasil: estágio atual, desafios e perspectivas. Revista de Empreendedorismo e Gestão de pequenas empresas. v.2, n.1, p. 3-21. 2013.

VergarA, S. C. Projetos e Relatórios de Pesquisa em Administração. 14a Ed. São Paulo: Atlas, 2013. 